\title{
La diversidad cultural y su impacto en el turismo comunitario de la región Andina
}

\section{Cultural diversity and its impact on community tourism of Andean region}

\author{
Esther del Carmen Mullo Romero' ${ }^{1}$, Martha Raquel Padilla Vargas ${ }^{1}$
}

凶Autorde correspondencia: emulloromero@gmail.com,martha_padilla79@yahoo.es

${ }^{1}$ Universidad de La Habana, Cuba.

Artículo original de la ponencia presentada en el IV CONGRETUR - "El impacto del turismo en el desarrollo del Ecuador", Universidad Central del Ecuador, 21 al 24 de noviembre de 2018

\begin{abstract}
Resumen
El artículo identifica el impacto de la diversidad cultural en el turismo comunitario de la región andina, así como también las prácticas del turismo comunitario desde la comunidad propuesto desde su propio enfoque cultural. El trabajo es una síntesis de revisión bibliográfica y aportes de las autoras para el uso y manejo sustentable de la diversidad cultural que existe en la región andina. Se enmarca en la investigación documental con un carácter descriptivo del objeto de estudio y fundamentado bajo los criterios de la investigación-acción, etnográfico y algunos aspectos teóricos de la historia local. El impacto que se le puede dar a la diversidad cultural de una región o sector, no sólo puede revivir, volver a poblar áreas rurales, despertar interés en una población apática, lograr cohesión social, sino que además, puede desencadenar actividades económicas y con ello mejorar los ingresos y la calidad de vida de la colectividad a través de un turismo responsable y qué mejor con las mismas comunidades que posibilitan el cambio cultural real con el visitante, en virtud que pueda profundizar en las tradiciones, costumbres y formas de vida del pueblo visitado. En los resultados se aprecia que la cooperación de instituciones para el desarrollo del turismo comunitario es muy importante ya que ayuda al desarrollo y dinamización económica de la comunidad, debido a que presenta gran variedad de productos como es su naturaleza, arqueología, la propia comunidad -vivencias-, la cultura de forma general.
\end{abstract}

Palabras clave: Diversidad, Cultura Comunitaria, Economía, Práctica Comunitaria, Intercambio Cultural.

\begin{abstract}
The article identifies the impact of cultural diversity on community tourism in the Andean region, as well as community tourism practices from the community proposed from its own cultural approach. This work is a synthesis of bibliographic review and contributions of the authors for the use and sustainable management of the cultural diversity that exists in the Andean region. The research is documentary with a descriptive approach of the object of study and based on the criteria of the action research, ethnography, and some theoretical aspects of local history. The impact, which can be given to the cultural diversity of a region or sector, cannot only revive, repopulate rural areas, arouse interest in an apathetic population, achieve social cohesion, but also it can trigger economic activities and with it to improve the income and the quality of life of the community through responsible tourism and even better with the same communities that make possible the real cultural
\end{abstract}


change with the visitor, so that the tourist can deepen in the traditions, customs, and ways of life of the visited town. The results show that the cooperation of institutions for the development of community tourism is very important because it helps the development and economic dynamism of the community since it has a variety of products such as nature, archeology, the community itself - experiences- and the culture in a general way.

Keywords: Diversity; Community Culture; Economy; Community Practice; Cultural Exchange

\section{Introducción}

En el mundo globalizado de hoy, la diversidad cultural está al orden del día. Se habla insistentemente de pluralismo cultural y multiculturalidad, de culturas híbridas y sincretismos culturales, del derecho a la diferencia y de las políticas culturales diseñadas para respetar la diversidad y promover el entendimiento mutuo entre culturas. La Declaración Universal sobre Diversidad Cultural, adoptada por la UNESCO (2001), afirma que la diversidad cultural como realidad de nuestro mundo debe expresarse en las políticas de pluralismo cultural para la inclusión y participación de todos los ciudadanos.

Aquí se presenta el desafío de cómo comprenderse e interactuar entre los diferentes grupos culturales que existen, de personas de diversas fronteras debido a la apertura de los mercados y al aumento en la frecuencia de visitas a un cierto lugar con la que los líderes empresariales de cualquier origen cultural se enfrentan a grupos conformados por personas de diferentes culturas (García, Sosa \& Medina, 2006). Varios estados han reaccionado aprobando nuevas leyes y aun constituciones que reconocen la diversidad de culturas, así como los derechos colectivos de dichas poblaciones. Este ha sido el caso, por ejemplo, de Guatemala, Nicaragua, Colombia, Ecuador, Perú, Bolivia, Chile y Brasil (Wade, 2000). "En su devenir histórico, el hombre ha logrado cambiar sustancialmente los modos de vivir y ha superado dependencias ancestrales con respecto a la Naturaleza" (Delgado, 2005). De ahí la importancia para que organizaciones y comunidades indígenas vean al turismo como medio de reivindicación y autogestión sobre sus territorios y recursos, que sirve para reclamar un lugar en el Estado y en el mercado, a manera de táctica turística (Gould, 1999).

$\mathrm{Y}$ es aquí, cuando se afronta el desafío de la diversidad cultural, en el que nos encontramos con presupuestos teóricos y conceptos sobre la diversidad cultural situados desde diferentes ópticas. Por ejemplo, puede hablarse de la edad, género, religión, valores nacionales, área geográfica donde vive, lugar donde nació o de la nacionalidad que se tiene, que no es más que aquella descrita en el pasaporte: la ciuda- danía, por lo tanto, podemos decir que la diversidad cultural es todo lo que le identifica al individuo. De este modo la diversidad cultural y su impacto en el turismo comunitario se ha venido planteando y replanteando, tanto formas y circuitos de turismo, hasta (lo más importante) cuestionarse por los sentidos sociales que tiene y que debe tener el turismo. Por esta razón se puede decir que este tipo de turismo promueve directamente el intercambio cultural a través de sus actividades con el visitante, mostrando las tradiciones, costumbres y formas de vida del pueblo visitado. El turismo comunitario incorpora los aspectos culturales en su quehacer, caracterizándose principalmente por el liderazgo que asume la comunidad al organizar programas con actividades turísticas con puesta de sus atractivos turísticos y en donde la misma es quien se beneficia de todo lo puesto en escena.

El trabajo es una síntesis de revisión bibliográfica y aportes del autor para la conservación de las costumbres y tradiciones que son la base de la identidad de los pueblos y un puntal fundamental para el uso y manejo sustentable de la diversidad cultural que existe en un país megadiverso como es el Ecuador.

En este sentido los objetivos que este trabajo persigue son:

- Identificar el impacto de la diversidad cultural en el turismo comunitario de la región andina.

- Analizar las prácticas del turismo comunitario desde la comunidad propuesto desde su propio enfoque cultural.

\section{Materiales y métodos}

El área de estudio comprende la región andina de Ecuador que está formada por 11 provincias a lo largo de la Cordillera de los Andes, distinguiéndose sus majestuosos valles, volcanes y nevados como el Pichincha, el Cayambe, el Cotopaxi, el Tungurahua o el Chimborazo; en esta región se encuentra Quito, que es la capital del país. Precisamente, la región andina es un área de gran importancia para el desarrollo del turismo comunitario, pues sus zonas rurales y la población en su mayoría indígena, permiten que ac- 
tividades turísticas vayan naciendo y se desarrollen.

En cuanto a los materiales y métodos, podemos indicar que se halla sustentada en la investigación acción, en un proceso de búsqueda, análisis y reflexión en la acción, partiendo de la experiencia y práctica cultural, la cual permite la construcción de conocimientos y transformación del entorno. Debe estar dirigida a satisfacer necesidades específicas y dar soluciones a problemas concretos. Se desarrolla ante una situación problemática concreta, real y requiere soluciones prácticas. Es un procedimiento reflexivo, sistemático, controlado y crítico, que permite descubrir nuevos hechos o datos, relaciones o leyes en cualquier campo del conocimiento humano, es también una indagación o examen cuidadoso o crítico, en la búsqueda de los hechos o principios, una diligente pesquisa para averiguar algo (Lanz, 1994). La presente también está enmarcada en la investigación documental y de campo, con un carácter descriptivo del objeto de estudio y fundamentado bajo los criterios de la investigación-acción, etnográfico y algunos aspectos teóricos de la historia local. Todo esto orientado y apoyado por teóricos, para ser difundidos, para promocionar los valores artísticos de la comunidad, que en su mayoría eran desconocidos por sus habitantes

\section{Resultados}

Luego del análisis de la información recolectada a través de las encuestas se resalta los siguientes resultados. Se aprecia que la cooperación de instituciones para el desarrollo del turismo comunitario es muy importante ya que ayuda al desarrollo y dinamización económica de la comunidad, debido a que presenta gran variedad de productos como es su naturaleza, arqueología, la propia comunidad-vivencias-, la cultura de forma general (Figura 1). El diseño del Plan Estratégico de Desarrollo de Turismo Sostenible 2006-2020 (PLANDETUR 2020) busca consolidar el turismo, con la participación del sector público bajo la rectoría del Ministerio de Turismo (MINTUR), el involucramiento activo de otras entidades gubernamentales, tales como consejos provinciales y municipios; y, la participación de los actores del sector privado y comunitario a través de las cámaras de turismo, los gremios y las comunidades locales.

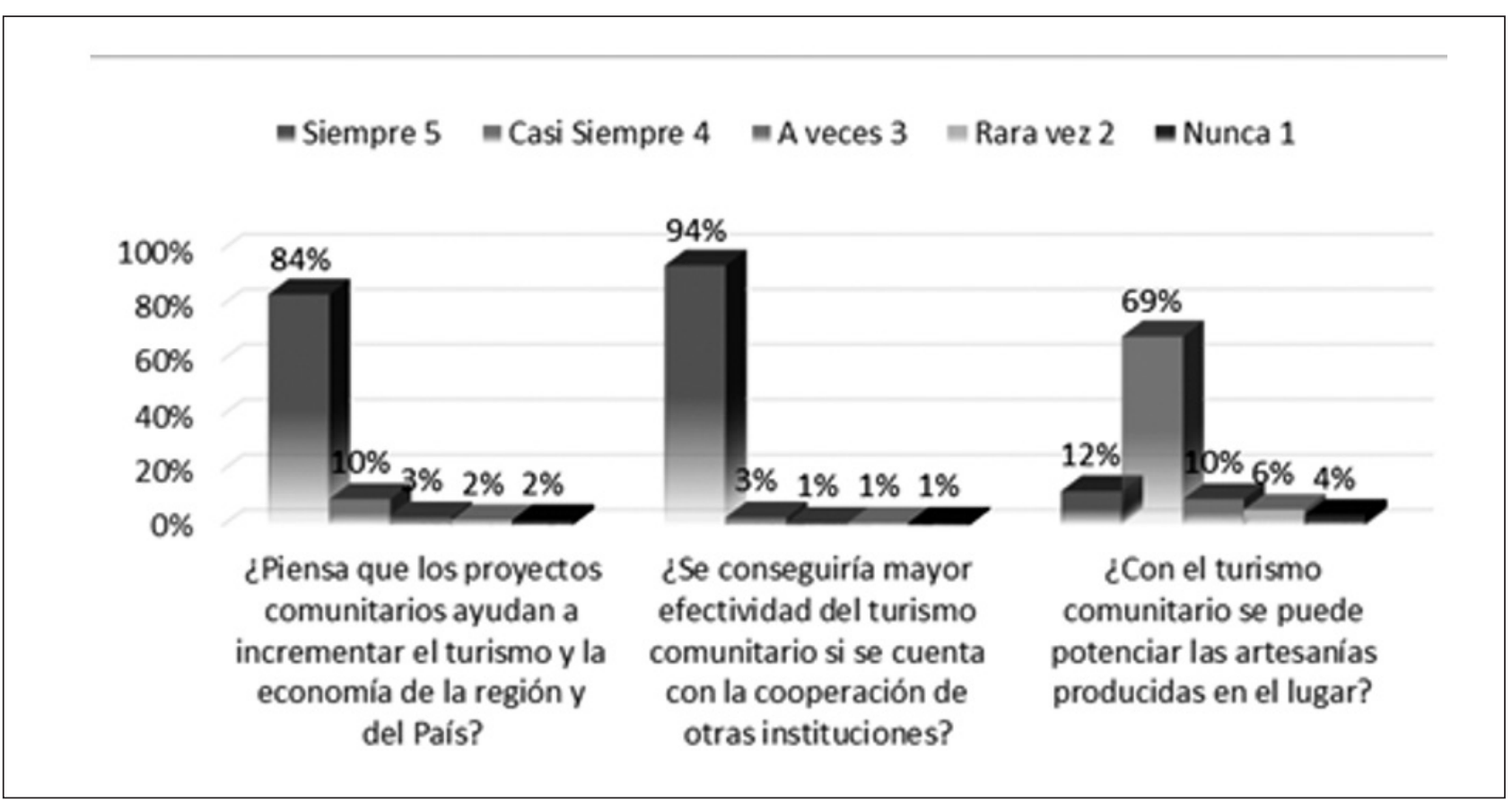

Figura 1. Resumen de resultados de la cultura tradicional presente en el turismo comunitario.

Por otra parte, la presencia de la diversidad cultural en el turismo comunitario sirve de desarrollo para las comunidades involucradas, ya que alcanzan a estrechar lazos en las relaciones laborales, así lo demuestran los resultados del siguiente cuadro en el que se observa un $78 \%$ cuando se habla de construir relaciones laborales productivas con otras culturas (Figura 2). En este sentido para muchas comunidades el turismo comunitario se ha convertido en un reavivamiento de sus espacios dentro del territorio, ayudando así, a que los pueblos indígenas se organicen y reclamen un lugar dentro del mercado turístico. 


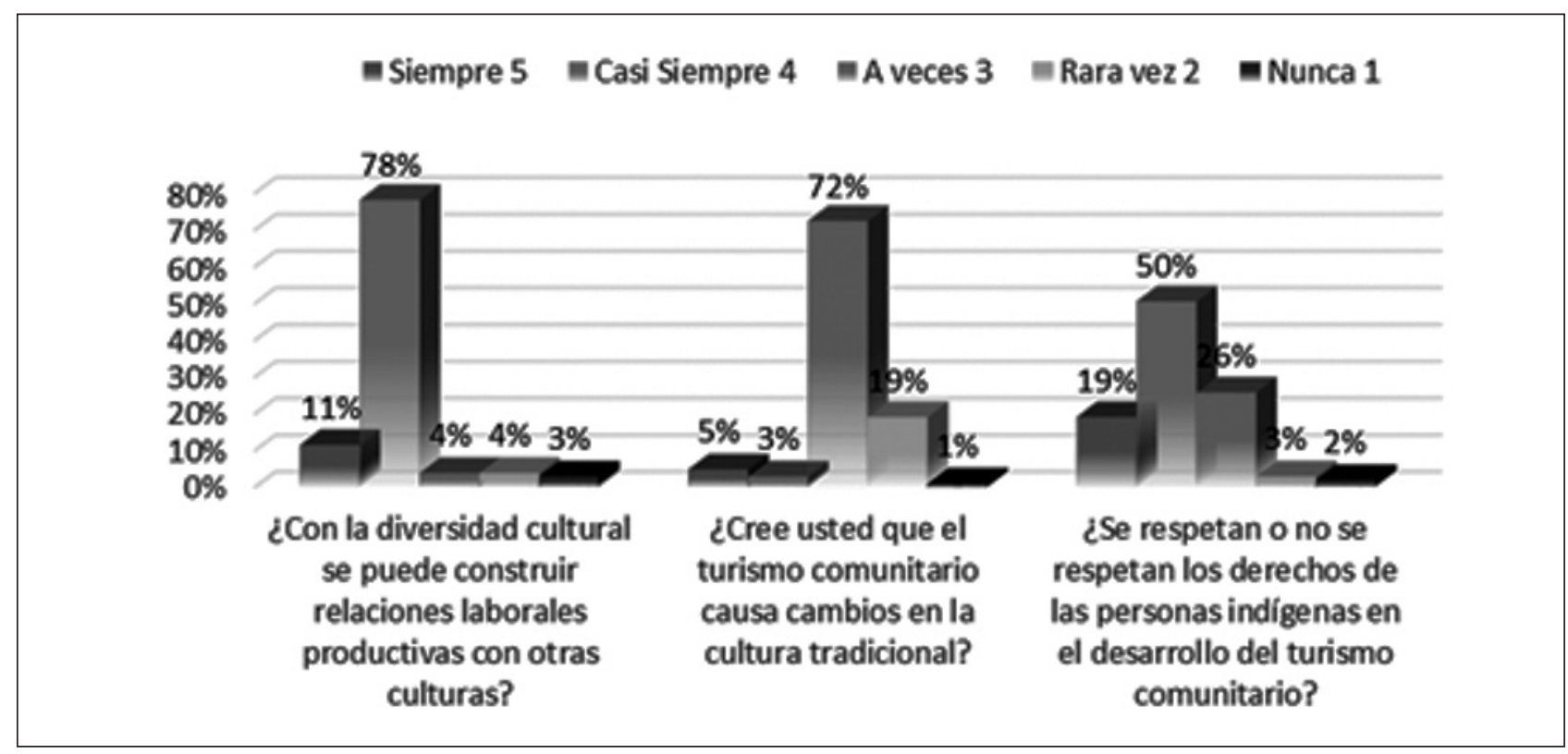

Figura 2. Resumen de resultados de la cultura tradicional presente en el turismo comunitario.

\section{Discusión}

\subsection{Las diversas interpretaciones de la diversidad cultural}

El término "diversidad cultural" ha sido objeto de diferentes interpretaciones y cambios derivados algunas veces de la historia, como el colonialismo, y de sutilezas lingüísticas o semánticas. Es posible distinguir dos acercamientos generales a la diversidad cultural: la diversidad "dentro" de los estados y la diversidad "entre" estados. Por ello la defensa de la diversidad cultural se basa "en el sano equilibrio que debe de lograrse con la ayuda de los diferentes grupos culturales que existen en el mundo, pero sin afectar a terceros o exceptuar alguna cultura" (CIESA, 2008). La excepción cultural pone de relieve la especificidad de los productos y servicios culturales, incluido el reconocimiento especial por parte de la Unión Europea, en su Declaración sobre la Diversidad Cultural.

Es importante destacar que la diversidad cultural no puede verse solo como una diferenciación (algo que se define en relación con otra cosa). La diversidad se manifiesta en situaciones concretas y necesita ser contextualizada, pues el sentido histórico de las "diferencias" redefine su propio sentido simbólico. Existen, en el mundo, diferentes organizaciones, como la UNESCO, que temen sobre la tendencia hacia una uniformidad cultural. Como prueba de ello se puede mencionar la desaparición de diferentes lenguas y dialectos, como por ejemplo, las lenguas de Francia, sin protección jurídica alguna que hagan valer el derecho y que, sobre todo, realcen su permanencia; también podemos tomar como ejemplo el aumento de la preeminencia cultural de los Estados Unidos a través de la manera en que distribuye sus productos de cine, televisión, cantantes, accesorios, ropa y productos de comida promovidos por los mismos medios de comunicación.

La diversidad cultural es la fuerza del desarrollo sostenible no solo para el crecimiento económico, sino para un complemento intelectual y moral. Así mismo, esta diversidad es un componente indispensable para reducir la pobreza y alcanzar la meta de un mejor desarrollo en la sociedad. Hablar de diversidad cultural es hablar de los reconocimientos que han impulsado los pueblos indígenas. La cultura es una parte fundamental de la sociedad y el mundo ya que se refiere a las formas en que se expresan los diferentes grupos en una sociedad que manifiestan su forma de pensar a través de diferentes modos de creación artística, producción y distribución de distintas ideas. Tenemos que imaginar una sociedad plural, multicultural, capaz de administrar la igualdad y la justicia en la diversidad cultural, una sociedad abierta y tolerante a las pluralidades que nos ofrecen las sociedades multiculturales y que desbordan las "fronteras culturales" y las antiguas fronteras sociales. Cuando se usa el concepto de diversidad, cada persona, cada grupo, cada comunidad necesita hablar de lo que es, de sus saberes, sus recursos, sus historias y proyectos, en suma, de su identidad. Porque lo diverso se define en relación consigo mismo 
y en relación con los otros, con los diferentes grupos de personas, tanto de un mismo sector como de un mismo país o continente.

La evolución de los conceptos, palabras y normas sirve para mirar los numerosos ejemplos de casos que en todo el mundo se multiplican en busca de darle un valor especial al conjunto de un territorio a partir de productos, prácticas, tradiciones y recursos que le son propios. Es importante entonces recordar que vivimos en un mundo de diversidad cultural que implica diferentes tipos de relaciones simultáneas, que permiten el intercambio de lugares de origen, idioma, comida, vestimenta, religión, incluso hasta trabajos, todos estos relacionados en una misma esfera de diversidad e igualdad.

La diversidad étnica-cultural, se podría conceptualizar como la herencia ancestral que da cuenta de la historia natural de la vida, por ejemplo de dónde venimos, quiénes somos, abarcando el conjunto de creaciones que distingue de los demás pueblos y da identidad a una nación, los valores espirituales, simbólicos, estéticos, tecnológicos y los bienes materiales que han aportado a la historia de la comunidad, estos alcances, según el Instituto Nacional de Patrimonio Cultural, se denomina patrimonio cultural, porque es propiedad de la nación llamada Ecuador (Instituto Nacional de Patrimonio Cultural, 2008).

\subsection{Identidad cultural}

"Todas las sociedades son heterogéneas culturalmente, ninguna cultura se desarrolla sin intercambios e interacciones culturales" (Perlo, 2006). La identidad cultural se puede decir que es el proceso por el cual se reconoce un determinado grupo social, y que constituye un atributo cultural, dentro de un determinado espacio.

Es importante tener presente también la distinción que se hace entre identidad cultural como las características que una persona o un grupo se atribuye para sentirse partícipe de una cultura concreta y la identidad de su cultura como referencia a las particularidades que se le podrían asignar de manera específica; es una cuestión sutil, pero una distinción muy importante, es a partir de allí que surgen las diferencias para hablar del derecho a la identidad cultural y el derecho a participar en la misma. Es decir, el derecho a la propia identidad cultural, al propio patrimonio y herencia cultural; y por otro lado el derecho al acceso y participación en la cultura como un bien primario, en el sentido de acceso, participación y disfrute de la cultura y la vida cultural. Así, la identidad se construye por las experiencias en distintas instituciones: la familia, la escuela, la religión, el trabajo, las cuales el individuo adapta, asume y recrea.

La diversidad cultural amplía las posibilidades de elección que se brindan a todos; es una de las fuentes del desarrollo, entendido no solamente en términos de crecimiento económico, sino también como medio de acceso a una existencia intelectual, afectiva, moral y espiritual que debe ser considerada como el conjunto de los rasgos distintivos espirituales y materiales, intelectuales y afectivos que caracterizan a una sociedad o a un grupo social y que abarca, además de las artes y las letras, los modos de vida, la manera de vivir juntos, los sistemas de valores, las tradiciones y las creencias.

Aunque existen diversas definiciones, en general, todas coinciden en que la cultura es lo que le da vida al ser humano: sus tradiciones, costumbres, fiestas, conocimiento, creencias, moral.

\subsection{Los pueblos y las culturas indígenas}

Si bien es cierto los pueblos indígenas constituyen hoy en día culturas profundamente transformadas por la conquista, la colonización, la evangelización, la asimilación lingüística y la subordinación al poder político estatal, los "pueblos testimonio" representan con su fuerte personalidad cultural una fuerza pujante que reclama el reconocimiento de sus derechos culturales y su legítimo lugar.

Según el Convenio 169 de la OMT los pueblos indígenas son considerados como tal por el hecho de descender de poblaciones que habitaban en el país o en una región geográfica perteneciente al país en la época de la conquista, colonización o establecimiento de las actuales fronteras estatales y conservan todas sus instituciones sociales, económicas, culturales y políticas o parte de ellas (art. 1) (OMT, OCDE, EUROSTAT, ONU, 2001).

Por otro parte, la cultura en la mayoría de los países de América incluye numerosas tradiciones de origen indígena, por lo que fortalecer a las culturas indígenas fortifica el propio ser nacional. Muchos países de América tienen un nombre indio (Canadá, México, Cuba, Haití, Jamaica, Guatemala, Nicaragua, Panamá, Guyana, Surinam, Perú, Paraguay, Uruguay y Chile). No se trata entonces de crear una utopía indigenista, sino darles su reconocimiento que no es otra cosa que legitimizar el hacer y pensar de dichos pueblos, facilitando el sitio que les corresponde en un proyecto global integrador de todos quienes lo conforman. El reto principal de estos pueblos es su reconocimiento como unidades políticas constitutivas del Estado (Colombres, 2006). 


\subsection{La diversidad y su impacto en el turismo comunitario}

Hay que reconocer el impacto y el valor que la diversidad cultural representa para el desarrollo de las personas y para el desarrollo de las sociedades, facilitando la inserción en el mercado laboral, como también un progreso en los procesos de desarrollo económico y social (OEI, 2002).

La diversidad cultural juega un papel importante en el desarrollo de un territorio, busca rentabilizar económica y socialmente el espacio local o lugar donde se desarrollan y se centran los diferentes tipos de actividades turísticas en que las personas viajan con la intención de distraerse en su descanso y que les permitan acercarse y comprender culturas distintas a las suyas, a tal punto que muchos pueblos y lugares del mundo, como es el caso del continente Europeo y de América Latina, han apostado por una revalorización de lo cultural, de lo identitario (recreando incluso nuevas identidades culturales) y patrimonial como eje de su propio desarrollo.

Para García (2002) "La identidad es el viejo territorio del patrimonio y no es de extrañar que entre los objetivos reconocidos por la mayor parte de actuaciones patrimoniales que se realizan en estos ámbitos, figure la (re) construcción de las identidades locales".

El impacto que se le puede dar a la diversidad cultural de una región o sector, no sólo puede revivir, volver a poblar áreas rurales, despertar interés en una población apática, lograr cohesión social, sino que además puede desencadenar actividades económicas y con ello mejorar los ingresos y la calidad de vida de la colectividad a través de un turismo responsable y qué mejor con las mismas comunidades que posibilitan el cambio cultural real con el visitante, en virtud que pueda profundizar en las tradiciones, costumbres y formas de vida del pueblo visitado.

De esta manera podemos considerar a la diversidad como un atractivo especial de cada país, localidad o comunidad. Para Fernández (2009) "Los atractivos (o atracciones), como su nombre lo indica, son los elementos atrayentes de los flujos turísticos. Se dividen, de forma general, en "cosas para ver", "cosas para hacer" y "combinaciones para ver-hacer". Se puede decir entonces que el turismo comunitario logra insertar en los mercados emisores, solo las dos últimas que tienen verdadero éxito y ha logrado que diversas comunidades del país lo vean como un mecanismo de desarrollo dinamizador de la economía y un medio de puesta en valor de sus elementos culturales.

Todo esto enmarcado en un conjunto de oferta de servicios de productos turísticos, que se colocan oportunamente en el mercado, que pueden ir desde lo agropecuario hasta actividades orientadas al turismo. Mientras las sociedades de los lugares se convierten en las sociedades de los flujos, parece como si los lugares se hubieran involucrado en una obra de construcción identitaria, que privilegia la dimensión local o ciudadana por encima de las nacionales, estatales y globales.

En el caso de los servicios de turismo, tiene una particular relación histórica con el patrimonio. De acuerdo con algunos autores, se podría hablar de turismo cultural desde la época griega y romana, y es el siglo XVIII el que estrecha la relación viaje patrimonio cultural, al aparecer los primeros museos públicos y lo que actualmente se conoce como recorridos turísticos culturales. En el siglo XIX aparecen las colecciones de guías de viaje y el concepto patrimonial abarca lo etnológico. En el siglo XX surge el turismo de masas y otras formas de turismo relacionado con lo patrimonial: ecológico, temático y activo y cultural.

\subsection{Turismo comunitario desde las comunidades}

El turismo comunitario en los actuales momentos, está siendo reconocido como una "marca internacional" y un elemento de creciente interés en la oferta y demanda turística de varios países, tanto es así que existen diversidad de experiencias de turismo comunitario en Asia, África, Canadá, Australia, Estados Unidos y Nueva Zelanda, en los países de habla hispana, desde México hasta Bolivia, pasando por Centroamérica y los países andinos, e incluso Argentina, encontramos tanto experiencias locales como federaciones nacionales y redes que agrupan dichas iniciativas y trazan su propio camino en el mundo del turismo (Ruiz et al., 2008).

Se señala al Ecuador como el país precursor, por el peso cuantitativo y cualitativo de sus experiencias de turismo comunitario. Así, desde los años ochenta, el turismo comunitario se ha convertido en Ecuador en una actividad estratégica para muchas comunidades ya que actúa como catalizador de diferentes procesos. De esta forma, el turismo comunitario se convierte en un campo estratégico de desarrollo social, económico y cultural, que parece encarar con éxito varias de las más acuciantes expectativas del mundo actual.

Así, para sus actores, "Turismo comunitario es toda actividad turística solidaria que permite la participación activa de la comunidad desde una perspectiva intercultural, manejo adecuado del patrimonio natural y valoración del patrimonio cultural, 
basados en un principio de equidad en la distribución de los beneficios locales" (FEPTCE, 2007). El turismo comunitario local se ha convertido en el nuevo activador de las políticas de desarrollo dando a conocer los estilos de vida, costumbres, tradiciones, festividades, historia, arquitectura y monumentos del lugar visitado. La propia definición de turismo comunitario subraya una forma de gestión diferente -comunitaria- sobre una actividad y recursos, lo que significa en algún grado la existencia de un espacio de organización autónoma, desde el cual vincularse al mercado y a otros actores (ONGs, organizaciones, Estado). Lo comunitario en Ecuador se concreta en instituciones (es decir, órganos político-administrativos) cuyos objetivos son regular la gestión de derechos, obligaciones, y reconocimiento del pasado, de la historia bajo la simetría de igualdad. La Organización Internacional del Trabajo (OIT) define al turismo comunitario como "Toda forma de organización empresarial sustentada en la propiedad y la autogestión de los recursos patrimoniales comunitarios, con arreglo a prácticas democráticas y solidarias en el trabajo y en la distribución de los beneficios generados por la prestación de servicios turísticos, con miras a fomentar encuentros interculturales de calidad con los visitantes" (Maldonado, 2005).

El caso de la región andina es paradigmático, pero en modo alguno único. Allí, individuos diferentes entre sí, organizados en unidades domésticas y grupos familiares, donde la propiedad privada de la tierra y la producción agraria se convierten en la base de sus economías, se dotan de una organización política desde la cual autogestionar recursos y tomar decisiones en una esfera donde prima la propiedad y el sentido colectivo: el turismo. El éxito del turismo comunitario se vincula de forma directa con los procesos de participación, de gestión y de repartos de los mismos comuneros de beneficios que proporciona esta estructura organizativa que garantiza el control efectivo del grupo sobre el turismo, lo cual genera un efectivo empoderamiento colectivo sobre recursos y territorios. De ahí que sea crucial entender este particular funcionamiento, donde la minga y la asamblea juegan un papel muy importante demostrando un modelo de organización único de su cultura (con distintos nombres según el lugar: asamblea, comité, etc.) pero es allí donde han surgido proyectos de turismo comunitario.

El turismo no deja de ser una actividad complementaria a las economías kichwas, basadas en las producciones agrarias que se orientan al mercado, pero sobre todo en el autoconsumo que pro- porciona el cultivo de la chacra. Pero, aun así, es lo suficientemente relevante como para constituir un acicate del proceso comunitario. Es en este sentido que cabe afirmar que el turismo construye comunidad (a la vez que la comunidad construye el turismo). Así los procesos de fortalecimiento de la comunidad a través de la actividad turística sólo son comprensibles desde la existencia de una organización que permite traducir al "lenguaje" comunitario el hecho turístico y viceversa, insertándose así en el mercado de forma específica y diferenciada.

Uno de los argumentos que actualmente resaltan el valor de la diversidad cultural es que crea un mundo más interesante, y porque otras culturas poseen modelos alternativos de organización social que puedan resultar útiles de adaptar a nuevas circunstancias. Esto suele mencionarse en relación con los pueblos indígenas, cuyos estilos de vida tradicionales proporcionan un modelo de relación sostenible con el entorno. Se acepta cada vez más que las actitudes de turismo comunitario y hacia la naturaleza son insostenibles y autodestructivas, por lo que los pueblos indígenas "pueden proporcionar modelos, inspiración y guía, para la tarea esencial de redefinir un nuevo orden mundial" (Kymlicka, 1996).

\section{Conclusiones}

La diversidad cultural es un concepto muy amplio que nos permite entender la naturaleza de los pueblos y su dinámica y aprender a coexistir con ella, reconociendo sus diversos aspectos, como la identidad, expresiones culturales, pueblos indígenas, desarrollos en muchos ámbitos, así como en el turismo en el cual demuestran un amplio desarrollo organizacional a través de sus leyes.

El turismo comunitario contribuye, de una $u$ otra manera, a que la diversidad cultural de cada pueblo no se pierda, se mantenga vigente, demostrando a la vez que son pueblos vivos y gestores de su propia identidad a través de un sistema autónomo que para ellos ha sido un mecanismo de organización.

La actividad turística en las comunidades ayuda al desarrollo de la economía del sector, así como a la construcción de relaciones laborales, las mismas que permiten que todos los involucrados de forma directa $o$ indirecta se vean beneficiados, de esta manera se reaniman las zonas deprimidas, se aumenta y garantiza la permanencia de formas de vida tradicionales, y se contribuye con más empleo y la reactivación del turismo, especialmente de los sectores más desfavorecidos en las comunidades. 


\section{Referencias}

CIESA - Centro de Investigaciones y Estudios Superiores en Antropología Social. (2008). La diversidad cultural (Marco conceptual): campaña nacional por la diversidad cultural de México. México: Centro de Investigaciones y Estudios Superiores en Antropología Social.

Colombres, A. (2006). Diversidad cultural y proceso civilizatorio. En (coord.) Ameigeiras, A., \& Jure, E. Diversidad cultural e interculturalidad. (pp. 317-328). Prometeo Libros, España.

Delgado, C. (2005). Hacia un nuevo saber. La bioética en la revolución contemporánea del saber. La Habana: Acuario Publicaciones.

FEPTCE - Federación Plurinacional de Turismo Comunitario del Ecuador. (2007). Manual de calidad para la gestión del turismo comunitario en el Ecuador. Quito: FEPTCE.

Fernández R. (2009). Principios, organización y práctica del turismo. Primera edición. La Habana: Editorial Félix Varela.

García, G. (2002). Caracterización y sustentabilidad de las unidades para la conservación, manejo y aprovechamiento sustentable de la vida silvestre en Campeche. San Francisco de Campeche, Campeche, México: El Colegio de la Frontera Sur.

García, M., Sosa, S., \& Medina, M. (2006). La incidencia de los rasgos culturales en los resultados del equipo multicultural. Trabajo de investigación. España: Departamento de Economía y Dirección de Empresas, Universidad de Las Palmas de Gran Canaria, España

Gould, K. (1999). Tactical tourism. A comparative analysis of rainforest development in Ecuador and Belize. Organization \& Environment, 12(3), 245-262.
Instituto Nacional de Patrimonio Cultural. (2008). El corazón del Ecuador, su patrimonio. Tríptico divulgativo.

Kymlicka, W. (1996). Ciudadanía multicultural. Una teoría liberal de los derechos de las minorías. Barcelona: Ediciones Paidós Ibérica S.A.

Lanz, C. (1994). El poder en la escuela: El método INVEDECOR como fundamento del currículo alternativo. Caracas: Edit-Art.

Maldonado, C. (2005). Pautas metodológicas para el análisis de experiencias de turismo comunitario. SEED: Documento de trabajo núm. 73. Ginebra: Organización Internacional del Trabajo.

OMT, OCDE, EUROSTAT, ONU. (2001). Cuenta satélite del turismo, recomendaciones sobre el marco conceptual. Luxemburgo, Madrid, París, Nueva York: Publicación de las Naciones Unidas.

OEI - Organización de Estados Iberoamericanos para la Educación, la Ciencia y la Cultura. (2002). Cultura y desarrollo. Obtenido de: https://www.oei.es/historico/cultura/cultura_desarrollo.htm

Perlo, C. (2006). Educación e interculturalidad. En: Diversidad Cultural e Interculturalidad. Buenos Aires: Prometeo Libros.

Ruiz, E., Hernández, M., Coca, A., Cantero, P., \& Del Campo, A, (2008). Turismo Comunitario en Ecuador. Revista de turismo y patrimonio cultural Pasos, 6(3), 399-418.

UNESCO. (2001). Declaración Universal sobre la "Diversidad Cultural". $31 \mathrm{C} /$ Resolutions + CORR.

Wade, P- (2000). Raza y etnicidad en Latinoaméri$c a$. Quito: Editorial Abya-Yala. 